\title{
Sand Eddies Induced by Cyclic Tilt of a Retaining Wall
}

\author{
Chien-Hsun Chen · Dimitrios Kolymbas
}

Received: date / Accepted: date

\begin{abstract}
The cyclic tilt of a retaining wall induces a peculiar motion in the backfill (sand), which exhibits closed trajectories (eddies). In this paper the motion of the backfill has been optically traced and analyzed by means of PIV (particle image velocimetry), also known as DIC (digital image correlation). The results are of importance for cyclically loaded structures (e.g, piles for off-shore structures) and can also serve to test numerical simulations of large deformation.
\end{abstract}

Keywords Particle image velocimetry · cyclic loading $\cdot$ cyclic response $\cdot$ large deformation $\cdot$ retaining wall.

\section{Introduction}

As first observed by BOBRYAKOv et al. [1], the cyclic tilt of a retaining wall induces large deformations in the backfill consisting of dry sand. CuÉLlar et al. [2] applied cyclic loading to a pile embedded in saturated sand and found out that soil grains mixed beneath the free surface. Both of them directly [1] and indirectly [2] observed the convective motion of grain flow in the backfill. To study this motion in detail, a model test apparatus was built at the Division of Geotechnical and

\section{C.-H. Chen}

Institute of Infrastructure, Department of Engineering Science, University of Innsbruck, Technikerstraße 13, A-6020 Innsbruck, Austria

Tel.: $+43 / 512 / 507-62385$

Fax: $+43 / 512 / 507-62399$

E-mail: Chien-Hsun.Chen@uibk.ac.at

D. Kolymbas

Institute of Infrastructure, Department of Engineering Science, University of Innsbruck, Technikerstraße 13 , A-6020 Innsbruck, Austria
Tunnel Engineering of the University of Innsbruck (see Section 2.1). The grain motion was traced by digital photographs and analyzed by means of PIV (see Section $2.4)$.

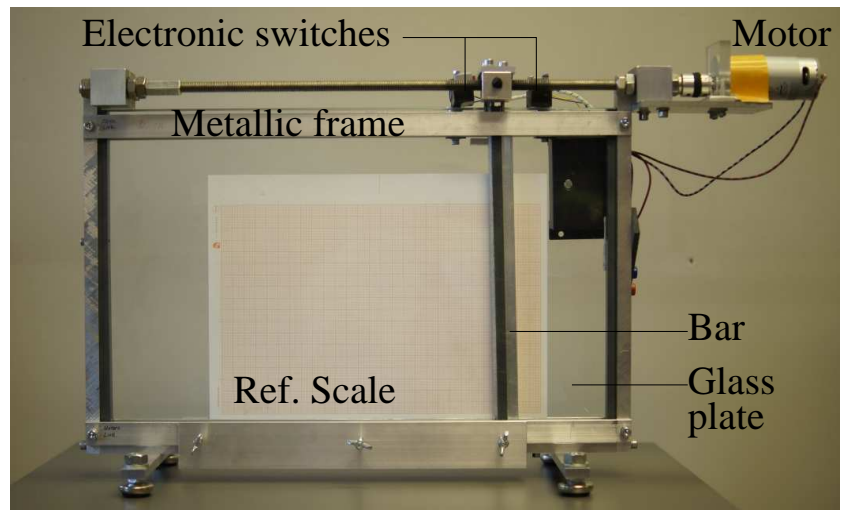

Fig. 1: Experimental apparatus.

We found that some grains track loops of quasielliptical form, as shown in Fig. 2. With a series of tests we analyzed the influence of the following parameters (see Section 2.2):

- amplitude of tilt, and

- geometry of the backfill.

\section{Experimental setup}

\subsection{Apparatus}

The apparatus is shown in Fig. 1. An aluminum bar serves as the retaining wall and is hinged at the bottom to enable tilt. The retaining wall was tilted by a 


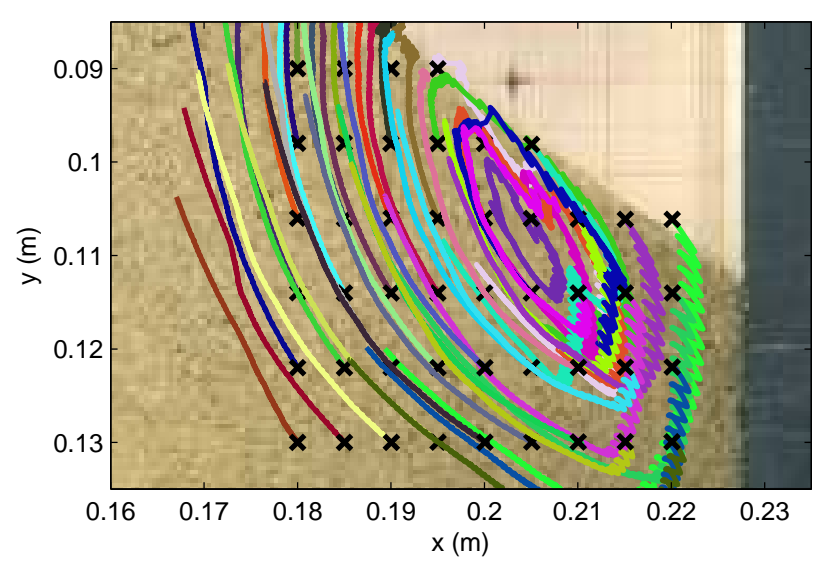

Fig. 2: Quasi-elliptical form of grain paths. Each colored line illustrates a trajectory starting at a location marked with ' $\times$ '.

motor and two electronic switches altering the directions of the drive of the motor. The bar and backfill are contained within two scratch-resistant glass plates fixed on a stiff metal frame with a spacing of $12 \mathrm{~mm}$. The tilt amplitude $\alpha$ of the retaining wall can be set by adjusting the positions of the electronic switches. For reference, a one-millimeter graph paper is affixed on the back-side glass. The backfill material consists of dry sand with a grain size distribution shown in Fig. 3.

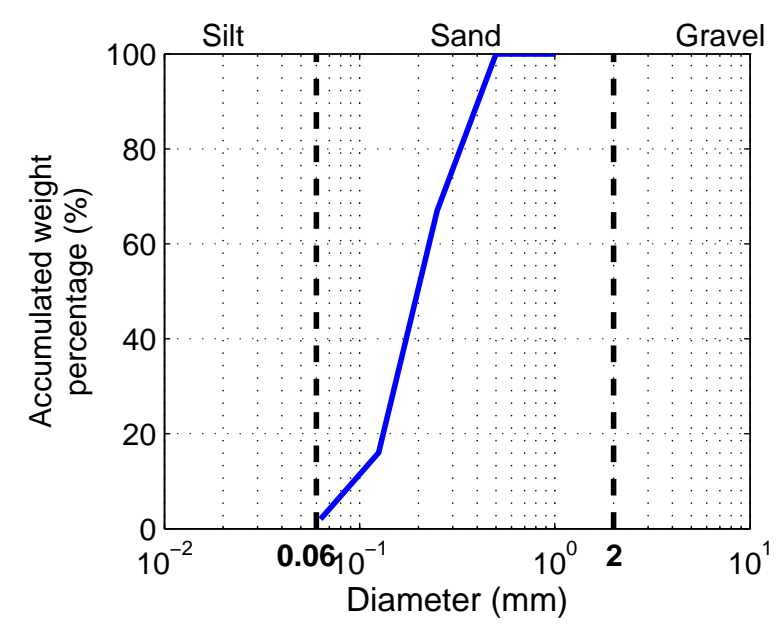

Fig. 3: Grain size distribution of test sand.

\subsection{Test series}

The conducted tests are summarized in Table 1 . The geometry parameters listed in Table 1 are illustrated in
Fig. 4. The tilt speed of the wall for $\alpha=1$ is 6 seconds per cycle and that for $\alpha=3$ is 18 seconds per cycle.

Table 1: Test series.

\begin{tabular}{ccccc}
\hline Name & $\begin{array}{c}\text { height } \\
h 2 / h 1 \\
(\mathrm{~cm})\end{array}$ & $\begin{array}{c}\text { surface } \\
\text { inclination } \\
\beta\left(^{\circ}\right)\end{array}$ & $\begin{array}{c}\text { tilt } \\
\text { amplitude } \\
\alpha\left(^{\circ}\right)\end{array}$ & $\begin{array}{c}\text { number } \\
\text { of } \\
\text { cycles }\end{array}$ \\
\hline Test 1 & $14.0 / 14.0$ & 0 & 3 & 60 \\
Test 2 & $16.1 / 7.8$ & 21 & 3 & 171 \\
Test 3 & $-/ 8.5$ & 34 & 1 & 236 \\
Test 4 & $14.3 / 14.3$ & 0 & 1 & 281 \\
\hline
\end{tabular}

*The initial density of sand can be hardly determined. Evaluating the total volume and the mass of sand, we found for Test 1 a value for density of $\rho=1.5 \mathrm{~g} / \mathrm{cm}^{3}$ and we assume that this value is valid for all tests.

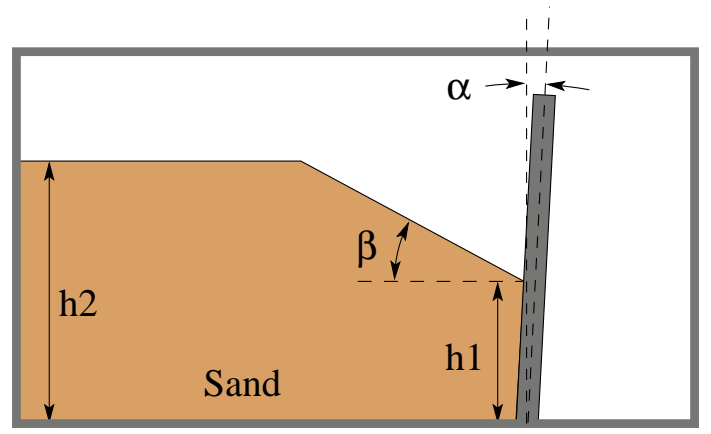

Fig. 4: Definition of the parameters in Table 1.

\subsection{Preparation of the backfill}

The preparation of the backfill is as follows: the wall is set to its vertical position with $\alpha=0^{\circ}$. Dry sand is pluviated using a funnel and keeping a constant falling height. The funnel is moved slowly back and forth to make a homogeneous backfill. The free surface was inclined by various angles, see Fig. 4 and Table 1 . The initial tilt direction, either inwards or outwards, was found to have no influence on the results.

\subsection{Particle image velocimetry}

In usual sand, the individual grains have various colors and form with their arrangement characteristic patterns. Comparing the patterns at times $t$ and $t+\Delta t$, we can identify clusters of grains and their motion. Of course, the deformation alters the form of each cluster. However, for small $\Delta t$ the alteration is small and the 
individual clusters can be identified by digital correlation analysis. This enables tracing the motion of the clusters and, hence, the motion of the grains. Consecutively tracing of this motion makes it possible to find the trajectories (paths) of grains over large time lapses. In our tests, a digital camera Sony $\alpha 35$ with a resolution of $3568 \times 2368$ pixels is used. The camera was placed in a distance of $0.50-0.60 \mathrm{~m}$ from the apparatus and the illumination was achieved with a halogen spotlight (500 Watt, J-Type, IP54) placed on the upper right or left corner behind the camera avoiding the direct reflection of the spotlight itself. The camera was leveled by waterlevels and oriented perpendicular to the glass plate. The open-source software PIVLab [3] was used to conduct the PIV analysis. It provides a friendly graphical user interface for image input and for PIV analysis, and has a variety of output formats and built-in functions for post-analysis. Besides, the user may easily mask out specific regions with arbitrary shapes in the images to avoid unnecessary calculations in the PIV analysis. The computation is also parallelized which allows an analysis with larger number of high-resolution images to be carried out in significantly shorter time.

\section{Results}

\subsection{Evolution of free surface}

The evolution of the free surfaces in our experiments is illustrated in Fig. 5. In Tests 1, 2 and 4, the subsidence of the free surfaces was observed. It was found to occur only near the wall. This area is termed "conical soil depression zone" by CheAng AND Matlock [4], and has been observed in their quasi-static cyclic tests. CuÉllar et al. [2] also observed such a phenomenon in their model test, along with two heaves at the edge of a conical soil depression zone on both sides of the pile in the loading direction. In addition, BOBRYAKOV et al. [1] and CuÉLLAR et al. [2] used the position of the free surface as a criterion to distinguish the motions of the backfill. To explain the hereafter used notion of global heave and local heave, we refer to Fig. 6. A global heave appears at the edge of the depression zone whereas a local heave indicates a temporary tiny one within the depression zone.

Tests 1 and 4 (Figs. 5a and 5b) started with a horizontal free surface, the same configuration as in CuÉLLAR et al. [2]. The tilt amplitudes were $\alpha=3^{\circ}$ and $\alpha=1^{\circ}$, respectively. Test 2 started with a surface inclination angle of $21^{\circ}$, similar to the experiment carried out by BoBryAKOv et al. [1]. In the early cycles of these experiments before the free surface subsided to a steady

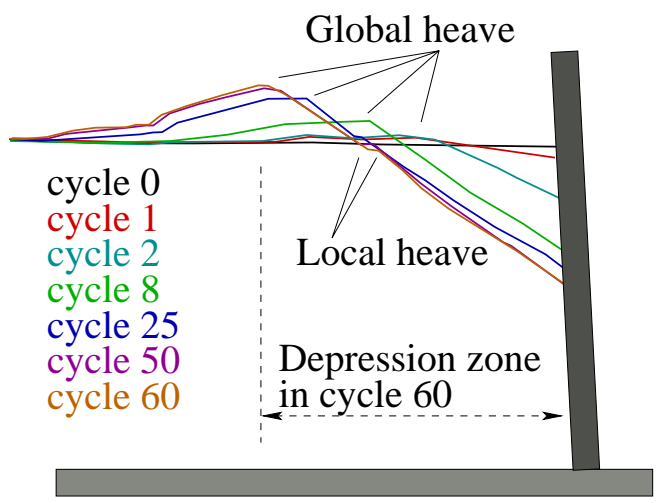

(a) Test $1\left(\beta=0^{\circ}, \alpha=3\right)$

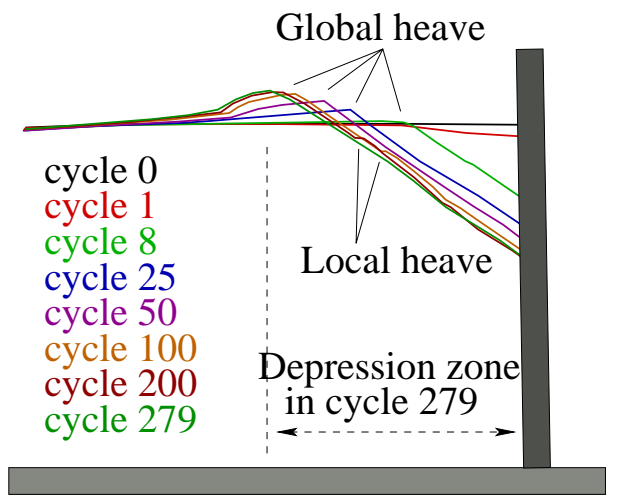

(b) Test $4\left(\beta=0^{\circ}, \alpha=1\right)$

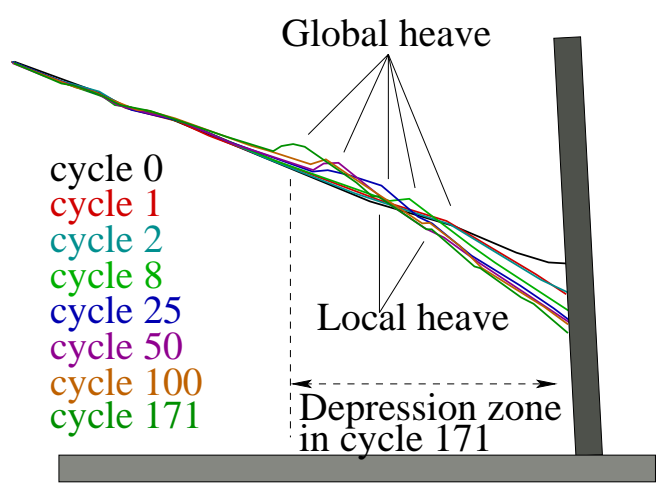

(c) Test $2\left(\beta=21^{\circ}, \alpha=3\right)$

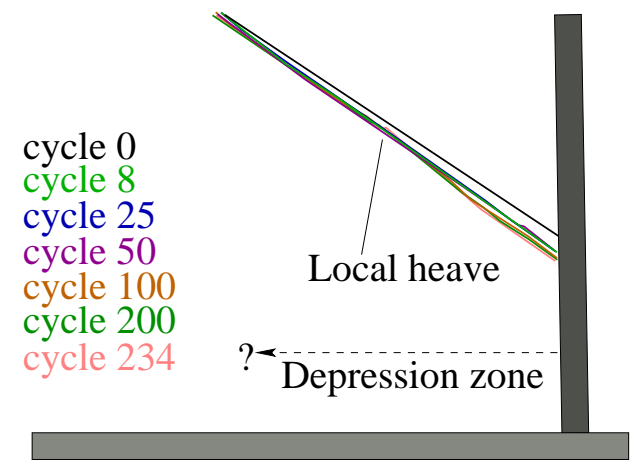

(d) Test $3\left(\beta=34^{\circ}, \alpha=1\right)$

Fig. 5: Evolution of the free surface. Free surfaces at various cycles are marked with different colors. 


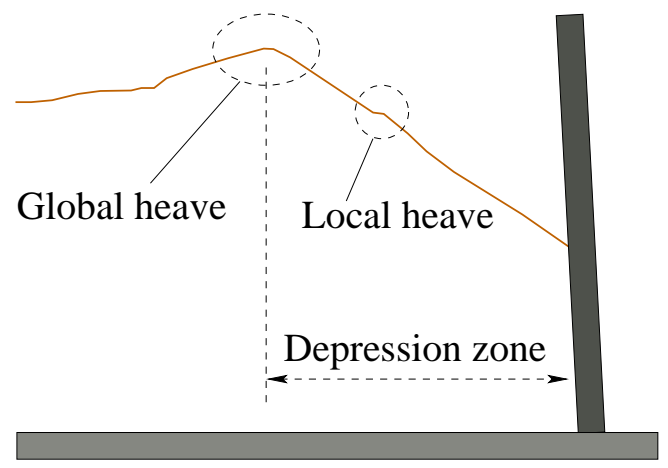

Fig. 6: Definition of global heave, local heave, and depression zone from a free surface.

position, the backfill slipped into the empty space created when the retaining wall tilted outward. Therefore, the surface near the wall dropped a bit, forming a slope. This motion corresponds to the failure mechanism of active earth pressure, as shown in Fig. 7a. When the retaining wall was pushed against the backfill, about $2 / 3$ of the total length of the surface close to the wall rose slightly and a global heave began to form. This type of movement is associated with passive earth pressure, as shown in Fig. 7b. The pressure response caused by the backfill acting on the wall can be found in BOBRYAKOV et al. [1].

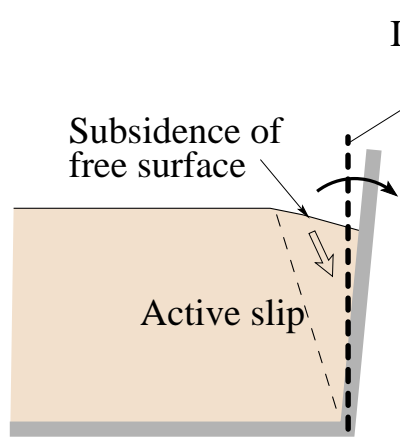

(a) Outward

\section{Initial position}

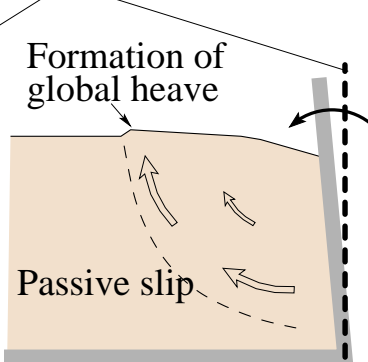

(b) Inward
Fig. 7: Illustration of the formation of slope and heave(s) induced by (a) outward and (b) inward wall tilts.

With increasing number of cycles, the surface inclination became steeper and the surface near the wall continued to sink until the surface reached a stationary position. At this position, the slope angle is $35^{\circ}$, measured by the average surface inclination over the depression zone. This angle is termed the critical inclination angle throughout this paper. Thereafter, from cycle to cycle, the global heave migrated progressively away from the wall to the left and became bigger (e.g. see cycles 8, 25, 50 in Fig. 5a and cycles 25, 50, 100 in Fig. 5b); the surface sank deeper. The results also show that the size of the global heave is proportional to the tilt amplitude.

After a number of cycles, the alteration of the surface in shape and position significantly retarded (e.g. cycle 25 in Fig. 5a and cycle 100 in Fig. 5b). In this situation, the surface reached a stationary position although minor changes continued over cycles, e.g. the migration local heaves, the appearance of new local heaves and the migration of the global heave. BOBRYAKOV et al. [1] used this phenomenon as a criterion to divide the motion of the backfill in their experiment into firstly a non-stationary phase, followed by a stationary phase characterized as "steady-state material density and unchanging specimen surface". The same phenomenon has also been observed by CuÉLLAR et al. [2], with the depression of the surface reaching a steady depth "within the first thousands of cycles". In [2], two distinct phases of deformation and grain displacement were defined using the stationary position of the subsidence surface: a densification-dominated phase and a convection-dominated phase. However, it should be noted that, based on our observations, the surface reaching its stationary position is a progressive process requiring many cycles.

On the contrary, the change in slope configuration is not significant in Test 3, as shown in Fig. 5d. The experiment started with a surface inclination of $34^{\circ}$. The evolution of the surface shows that the slope inclination increased and reached $35^{\circ}$, and the surface dropped slightly. In addition, no global heave was formed throughout the experiment, since the material on the upper slope slipped when the slope reached the critical inclination angle of $35^{\circ}$, preventing the formation of a global heave.

It is noted that Figs. $5 \mathrm{c}$ and $5 \mathrm{~d}$ illustrate a minor influence of the cyclic tilt on the evolution of the free surface for inclined surface, when compared with Figs. $5 \mathrm{a}$ and $5 \mathrm{~b}$ for horizontal surface. This is due to the fact that, in Tests 1 and 4, more material is placed in the depression zone, compared with Tests 2 and 4, as illustrated in Fig. 8. As the free surface in the depression zone tends to reach the critical inclination angle from cycle to cycle, for the case with an initially horizontal free surface, the volume of material behind the wall that is pushed away from the wall and merges with the material behind it is larger, when compared with the cases starting with an inclined free surface. As a result, the cyclic tilt has a more significant influence for tests starting with a horizontal free surface.

In all experiments, one or two local heaves periodically appeared after the free surface reached its station- 
(a)

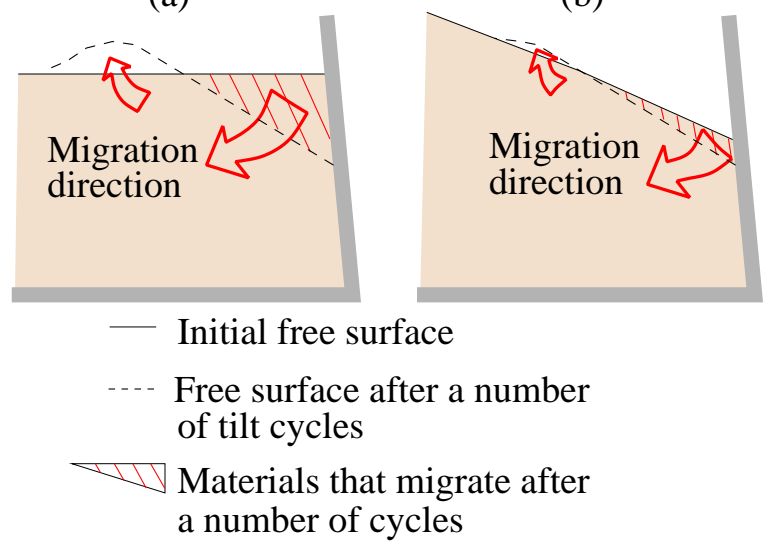

Fig. 8: Influence of the cyclic tilt on the evolution of the free surface for (a) horizontal surface and (b) inclined surface.

ary position. After their appearances, they migrated slightly and slowly towards the wall. It was observed that a local heave temporarily disappeared when the grains forming the local heave rolled down the slope, and re-appeared in the next cycles thereafter. In addition, before the material rolled down, the slope right at the local heaves, inclined by $37.5^{\circ}$ to the horizontal, is larger than the critical inclination angle $\left(35^{\circ}\right)$. Both global and local heaves are found to associate with the appearance of passive slip surfaces. Their periodical appearances are investigated and will be presented in the next sections.

CUÉllar et al. [2] observed a densification of the material in the "densification-dominated phase" by evaluating the volume change of the specimen. In all our experiments, the heaved volume is also clearly less than the subsided volume, and this proves that the densification in sand also took place before the surface reached its stationary position.

\subsection{Trajectories and eddies}

Sand eddies are elliptical trajectories of a spiral or a closed loop. Their positions, shapes, circulation directions, and the time of occurrence are described in this section.

\subsubsection{Shape and position of eddies}

Trajectories obtained after the free surfaces reached their stationary positions are shown in Fig. 9.

In these figures, eddies are elliptical in shape and are located below the subsided free surface adjacent to the wall. If we roughly describe the orbits of the particles

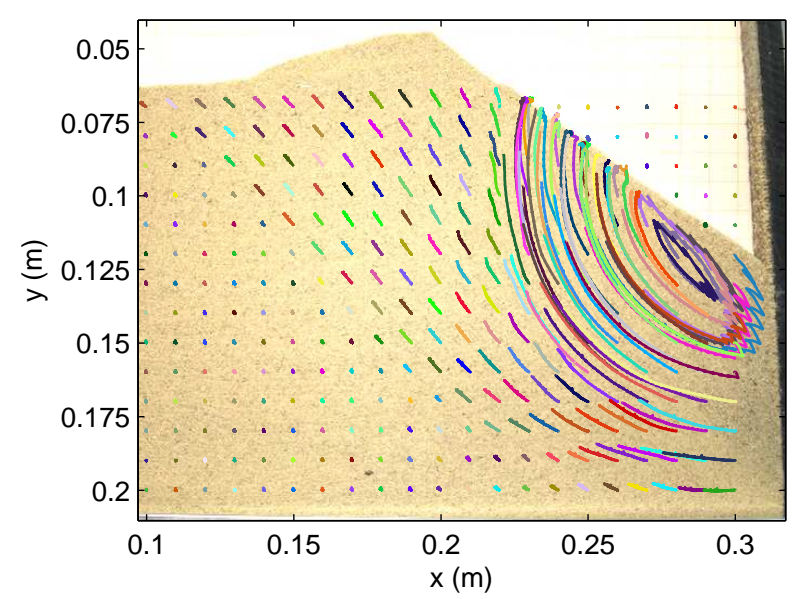

(a) Test $1\left(\beta=0^{\circ}, \alpha=3\right)$

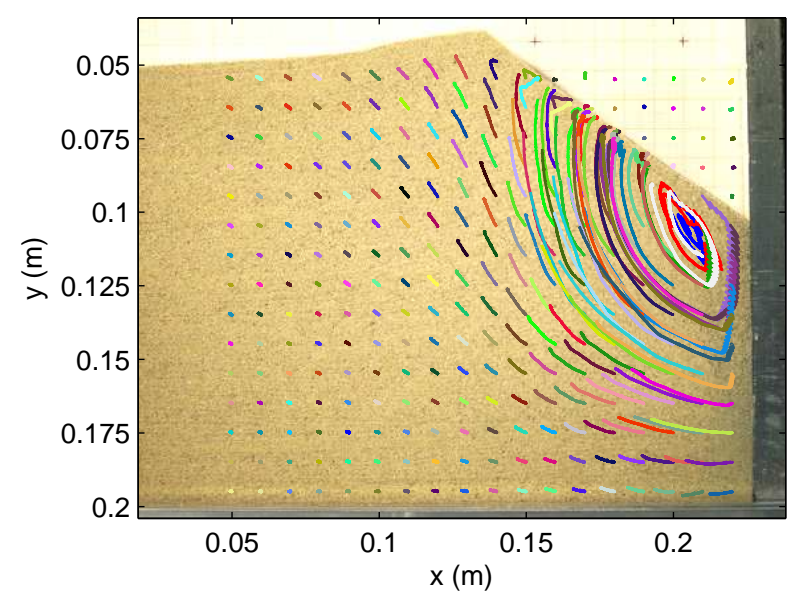

(b) Test $4\left(\beta=0^{\circ}, \alpha=1\right)$

Fig. 9: Trajectories during (a) cycles 25 to 60 and (b) cycles 50 to 100. Background photographs taken at the beginning of these cycles. (continued)

as ellipses, then we observe that the halve axes of these ellipses do not associate with the orientations of any geometries of objects in our experiments. Closer views of the eddies, as shown in Fig. 10, reveal that the elliptical shape is not smooth and the size of eddies varies. Moreover, eddies do not always have a closed loop. For instance, the trajectories plotted in Fig. 10a marked by green and in Fig. 10b marked by red have the form of an outward spiral. Such outward spiral eddies are observed in all experiments. However, inward-spiral eddies, such as those in Fig. 10b marked by purple and blue, are also present.

The slip surface was identified by plotting the velocity field occurred at the 51th outward cycle of the wall (Fig. 11a), along with the trajectories from cycle 50 through 100 (Fig. 11b). The active slip surface is found 


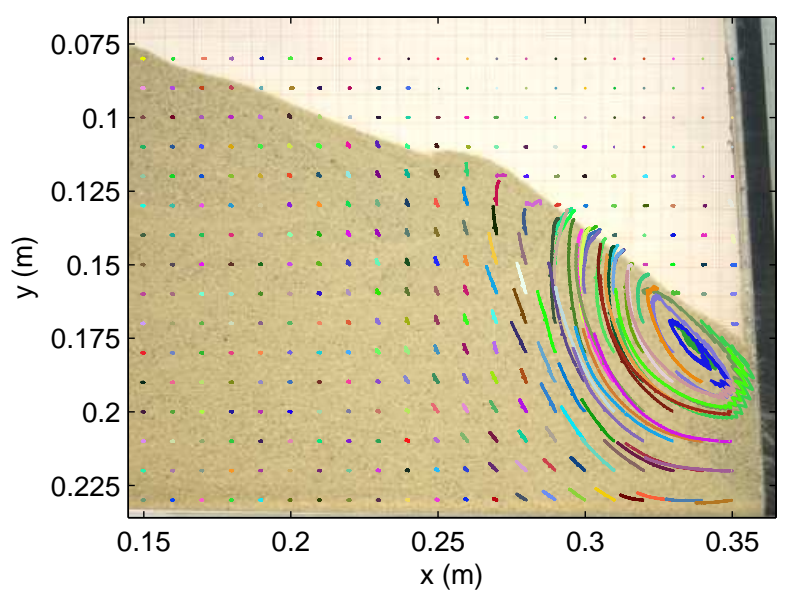

(c) Test $2\left(\beta=21^{\circ}, \alpha=3\right)$

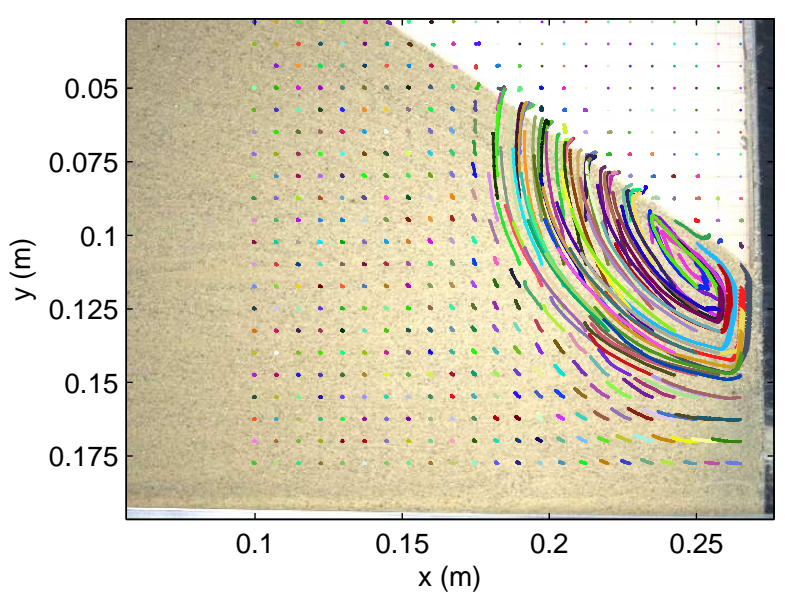

(d) Test $3\left(\beta=34^{\circ}, \alpha=1\right)$

Fig. 9: (continued) Trajectories during (c) cycles 50 to 200 and (d) cycles 50 to 200. Background photographs taken at the beginning of these cycles.

to cross the eddies and the surrounding trajectories. In addition, as shown in Fig. 12, trajectories below (or on the left side of) the active slip surface are smoother compared to those above (or on the right side of) it; and irregular zigzag trajectories are only found above the active slip surface (Fig. 12). These large curved zigzags result from the fact that the motion of the grains located on the right side of the active slip surface is influenced by the alternating tilt directions of the wall. The zigzag patterns hence are in sync with the cyclic tilt of the wall. The curved zigzags indicate that grains on the right side of the active slip surface move generally toward the lower-right direction. The grain motion on the left side of the active slip surface is insignificant when the wall tilts outwards, leaving the accumulated displacement being mainly due to the motion occurred

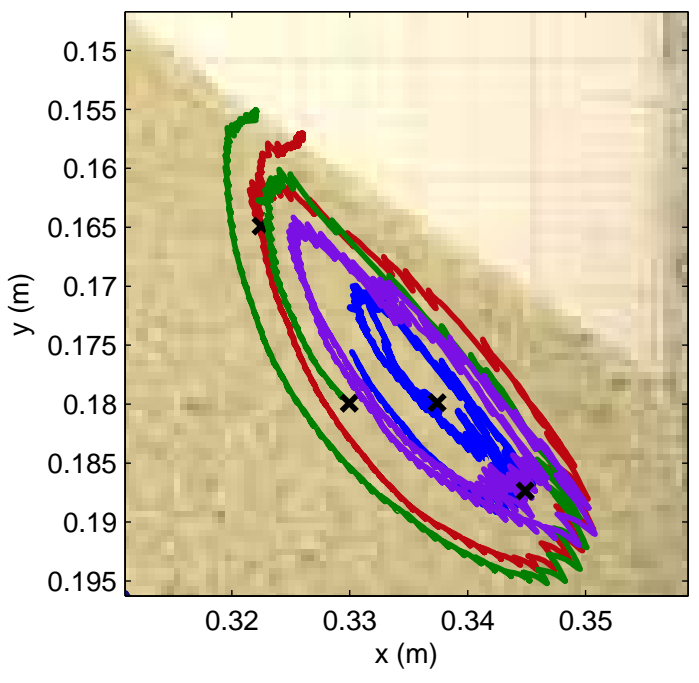

(a) $\alpha=3^{\circ} ; \beta=24^{\circ} ;$ Cycle $50 \ldots 150$

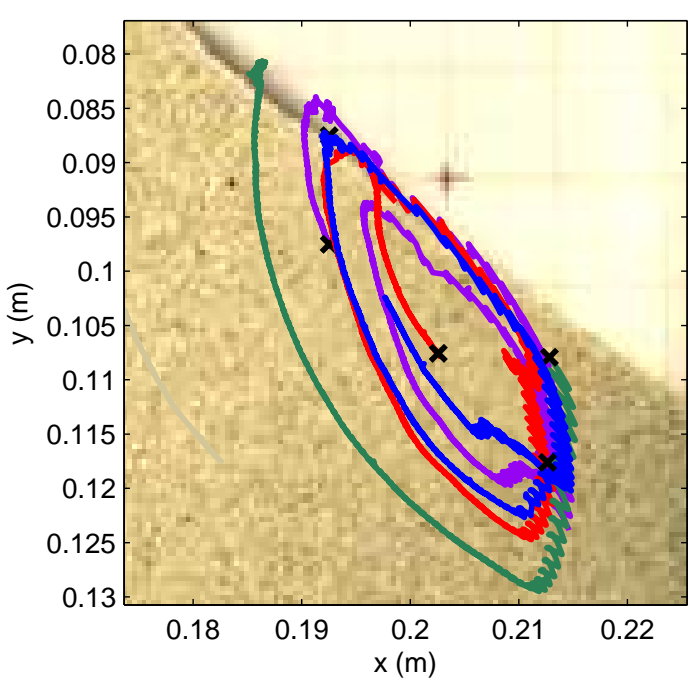

(b) $\alpha=1^{\circ} ; \beta=0^{\circ} ;$ Cycle $50 \ldots 200$

Fig. 10: Examples of various sand eddies in (a) Test 2 (background at cycle 50) and (b) Test 4 (Background at cycle 200). The origins of paths are marked with ' $x$ '.

during inward tilts of the wall. Trajectories on this side, therefore, turn out to be smoother. These trajectories are indicating that the grains migrate to the upper left.

As a result, an active slip surface can serve as a border which distinguishes smooth from less smooth trajectories. Intersections of active slip surfaces and trajectories are defined as "smoothness transition points, e.g. the hollow dots marked in Fig. 12. Defining the 


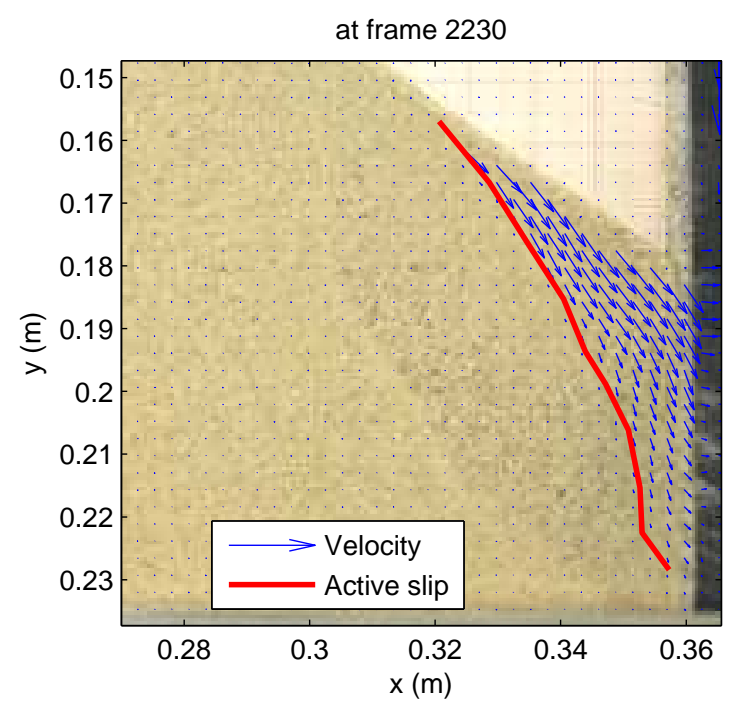

(a) At cycle 51

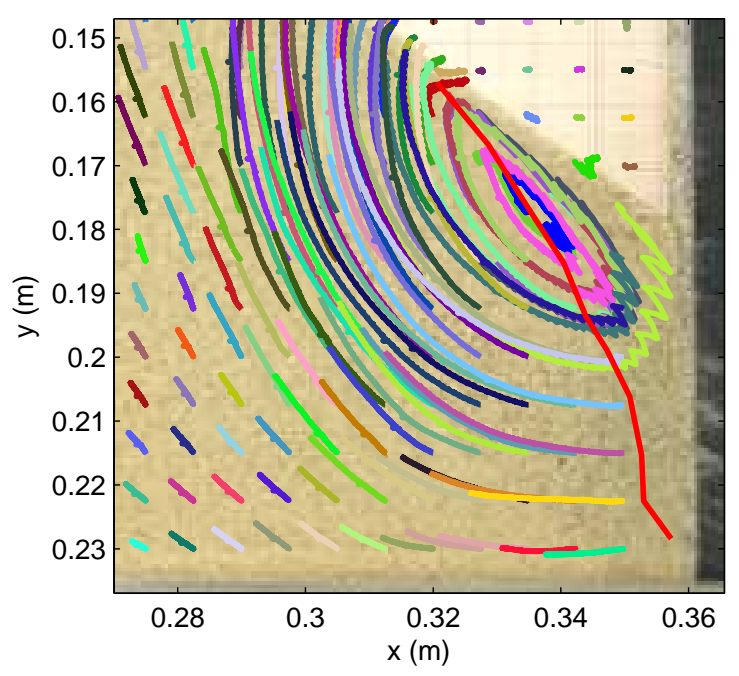

(b) Line construction

Fig. 11: (a) Active slip surface determined using the velocity field at cycle 51 while the wall was tilting outwards. (b) Comparison of the active slip surface and trajectories plotted during the cycles 50 to 100 in Test $2\left(\alpha=3^{\circ}, \beta=21^{\circ}\right)$.

smoothness transition points allows positioning of eddies in such experiments, since these points are situated on an active slip surface. If an eddy is pictured as an ellipse, the smoothness transition points are located around the antipodal points of the ellipse and the major axis of the ellipse shall approximately coincide with the active slip surface. This also explains the orientation of all eddies in Fig. 9.

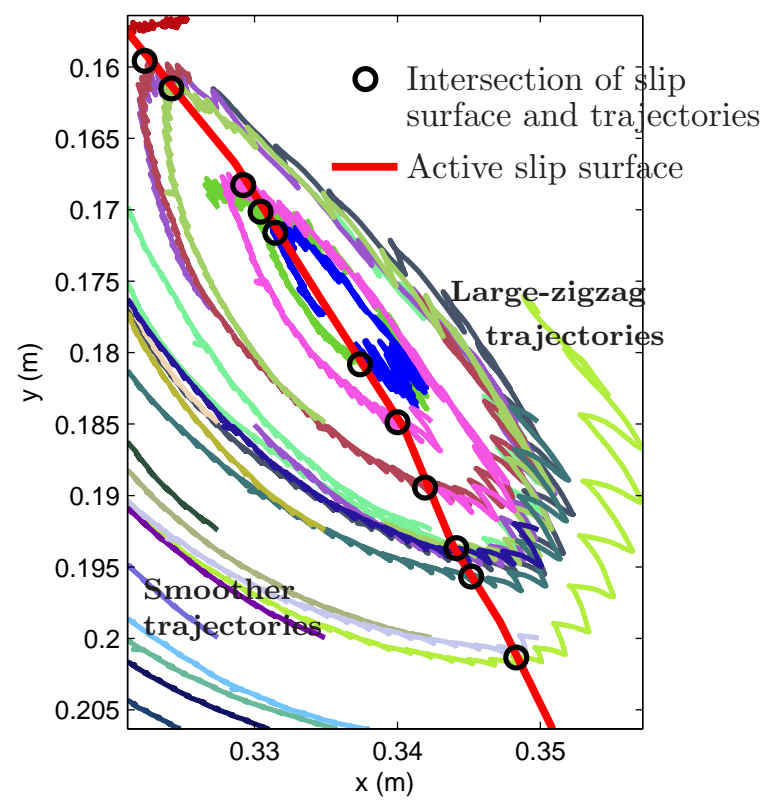

Fig. 12: Close-up view of Fig. 11b with the background removed. The trajectories above the active slip surface are large zigzags which are less smooth. On the contrary, the trajectories below the slip surface are smoother.

Information found in Fig. 12 helps to discover the area in which eddies occur, as illustrated in Fig. 13. The area, marked by green (Fig. 13a), is enclosed by the free surface, the trajectory which is below the active slip surface passing the intersection of the free surface and the active slip surface, and a boundary enveloping the zigzag trajectories (marked by a black dashed line).

\subsubsection{Zoning and passive slip surfaces}

CUÉLlar et al. [2] indicated that the movements of the grains possess different characteristics depending on where the grains are. They defined two domains based on the profile cutting vertically into the soil specimen along the loading direction (Fig. 14): (a) The "convected domain" where the convective flow of grains takes place. (b) The "static domain" where grain displacements are less significant. Between the two domains, there exists a thin transition band.

Different characteristics in grain motions, by means of trajectories, are also observed in our results. Take Test 1 as an example, the zoning based on the characteristics of trajectories is shown in Fig. 15a and three domains are defined. Domain I corresponds to the convected domain and Domain II and III correspond to the static domain defined by Cú́Llar et al. [2] (Fig. 14). 


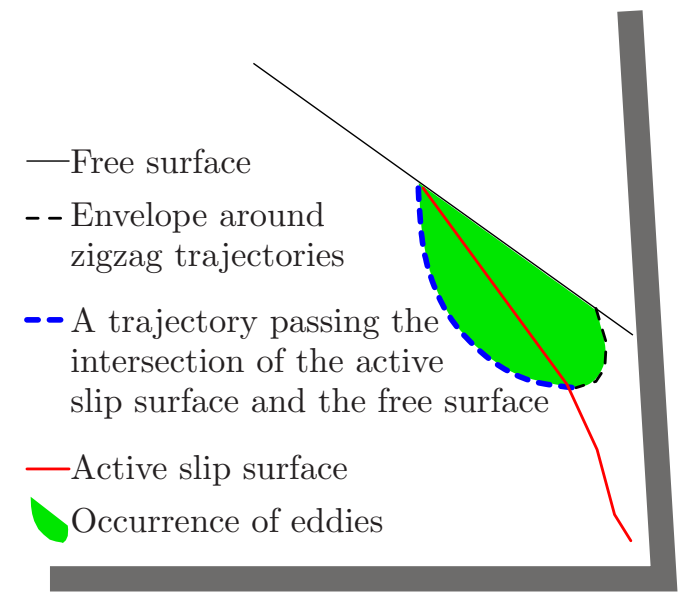

(a)

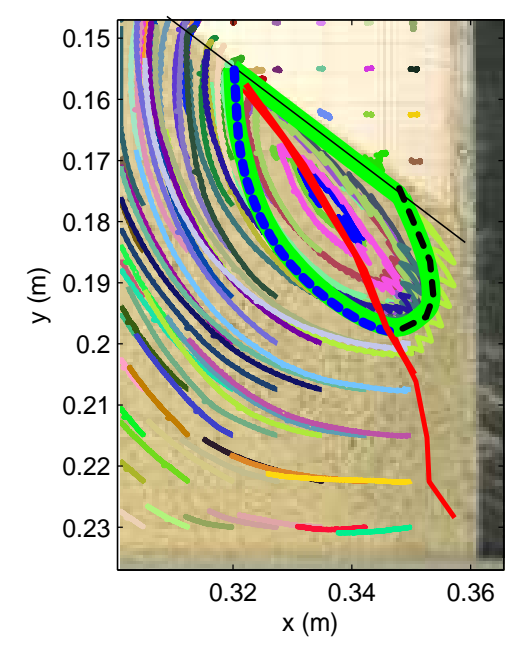

(b)

Fig. 13: (a) Area in which eddies take place. (b) Example: Test 2 (Fig. 11b).

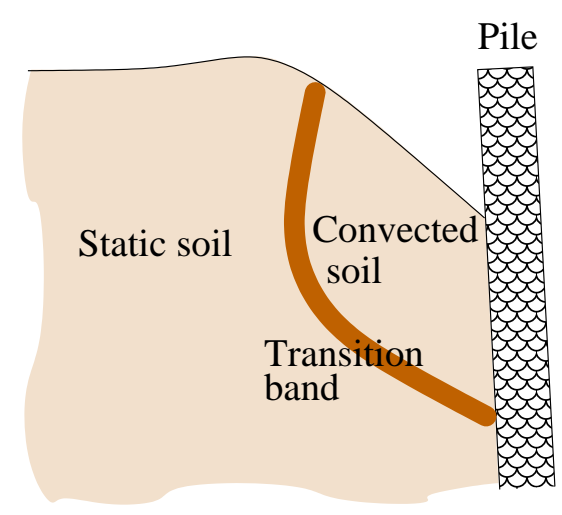

Fig. 14: Illustration of soil domains defined in [2].

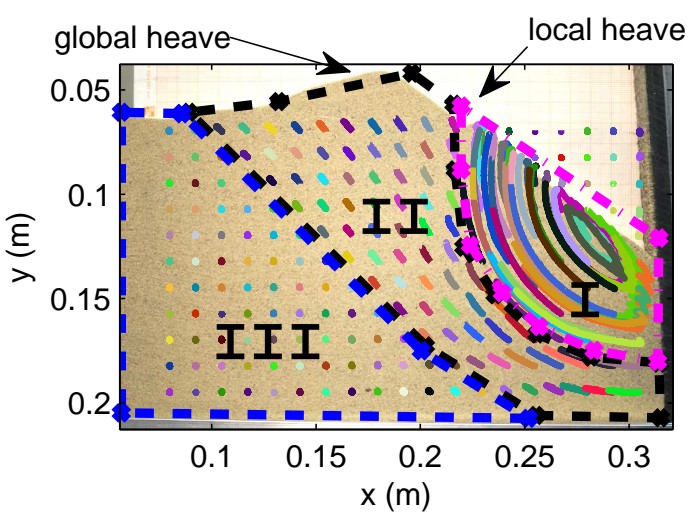

Fig. 15: Zoning by means of trajectories plotted from cycle 25 through 50 in Test 1.

In domain I, eddies, large-zigzag trajectories and smoother trajectories are present. Grains on eddies will migrate elliptically until they leave the eddies, ending up with re-appearing on the surface. This might explain the re-appearance of the red grains on the soil surface in [2]. In their experiment, the red grains were initially allocated on the free surface. They sank beneath the surface within the depression zone and re-appeared again on the free surface after a number of cycles. Trajectories in domain II are significantly shorter than those in domain I, suggesting that only minor displacement is accumulated, inducing the formation of the global heave. The size of domain II is associated with the initial surface inclination angle (compare plots in Fig. 15). In particular, the smaller $\beta$ is, the wider domain II will be. It is noted that domain II can be barely recognized in Test 3 (Fig. 9) that started with a surface inclination angle of $34^{\circ}$. In domain III, the dot-like trajectories indicate little to no displacement. That explains the negligible alteration of the free surface on top of this domain. The evolution of the domains in size and shape throughout the experiments as well as the formation and the migration of global heaves can be reconstructed by the information provided in section 3.3, as passive slip surfaces are natural borders of domains.

According to our results (see section 3.3) and the observation made by BOBRYAKOV et al. [1], the border of domains I and II migrates from cycle to cycle as will be shown in section 3.3.

\subsubsection{Circulation directions of eddies}

Fig. 16 qualitatively illustrates the evolution directions of the trajectories based on Fig. 9. The evolution directions of the trajectories above the active slip surface are opposite to those below the active slip surface. As a result, the circulation directions of the eddies are clock- 
wise (in the case that the cyclic retaining wall is on the right side). This is valid for all tests we have carried out.

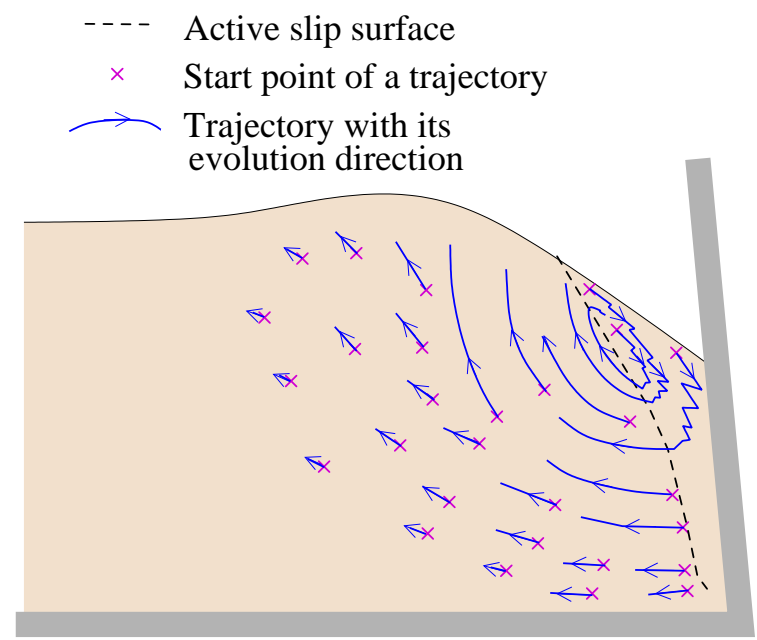

Fig. 16: Evolution directions of the trajectories.

\subsubsection{Time of occurrence of eddies}

CuÉllar et al. speculated that “..., it is possible that some convective grain migration already takes place simultaneously with the densification during the first loading cycles ...". This speculation is confirmed by our observations that eddies occurred in the beginning of all experiments and the positions of the eddies during an experiment are related to the evolving positions of the free surface adjacent to the wall. For instance, in Test $1\left(\beta=0^{\circ}, \alpha=3^{\circ}\right)$, as shown in Fig. 17a, during the starting cycles 1 to 11 , the eddies were tracked; as well as in Fig. 17b during the starting n 1 to 19.

\subsection{Evolution of passive slip surfaces}

The repeated process of the appearance of new passive slip surfaces and their migration towards the wall has been observed by BoBRYAKOv et al. [1]. In particular, after the appearance of a new slip surface, it shifts towards the wall and disappears at a certain position. During the course of its migration, another new passive slip surface is formed at a distance behind the existing one. Such repeated appearance of passive slip surfaces was also observed in domain I in our experiments, as shown in Figs. 18, 19 and 20. In addition, these figures also suggest that with a smaller tilt amplitude $\left(\alpha=1^{\circ}\right)$ (a) more cycles are required before the first passive slip surface became visible and (b) the presence of a passive

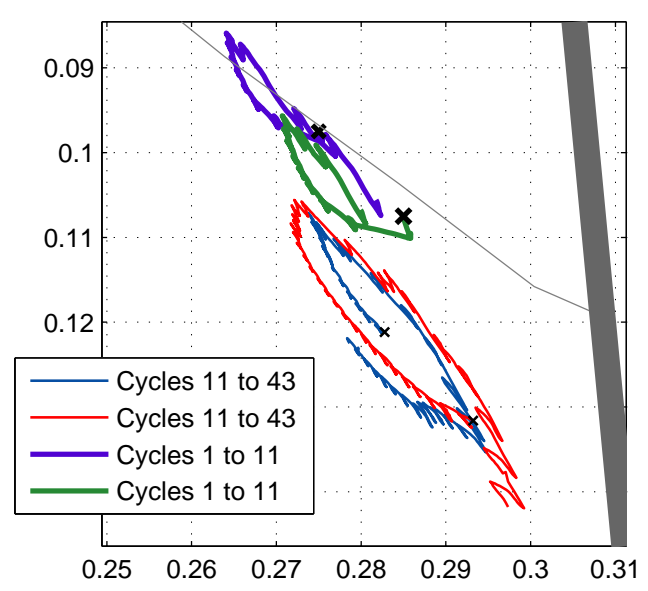

(a) Test $1\left(\beta=0^{\circ}, \alpha=3^{\circ}\right)$

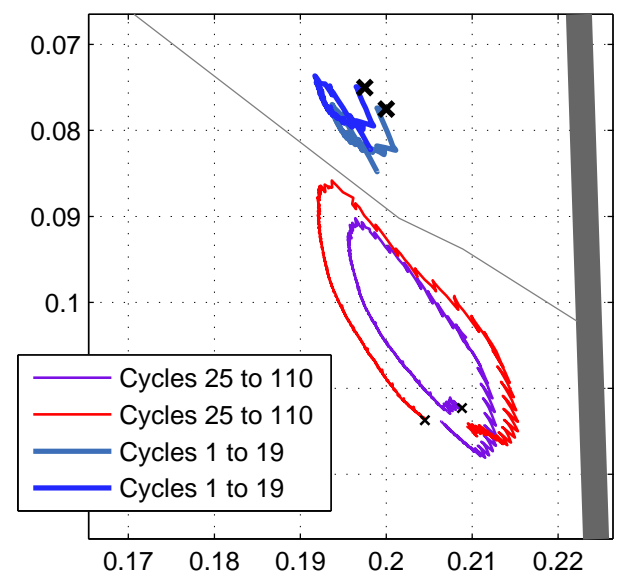

(b) Test $4\left(\beta=0^{\circ}, \alpha=1^{\circ}\right)$

Fig. 17: Eddies formed during various cycles in (a) Test 1, with surface captured at cycle 13, (b) Test 4, with surface captured at cycle 107.

slip surface lasted more cycles. It should be noted that an A-surface denotes a passive slip surface occurring in domain I (e.g. Fig. 15c) where the significant movements of grains to the up-left takes place. A B-surface denotes a slip in domain II where minor motion occurs. In case more than one surface is present, the sequence of occurrences of slips is stated by a number, with a greater number denoting a later appearance (e.g., A2surface occurs later than A1-surface).

No apparent trend of evolution of B-surfaces was noticed among experiments. Each stayed more or less at the same position during its presence. It is noted that B-surfaces were identified in Test $1\left(\beta=0^{\circ}\right)$ and in Test $4\left(\beta=21^{\circ}\right)$, but no B-surface occurred in Test 3 
$\left(\beta=40^{\circ}\right)$. This might result from the fact that domain II is very thin in Test 3, as shown in Fig. 9. In addition, B-surface was not exhibited at all times in Test 4. A missing border between domains II and III indicates that no clear slip surfaces were present, implying gradual changes of the velocity fields in direction and magnitude.

\subsection{Evolution of void ratio}

The PIV evaluation program yields the displacement vectors $\mathbf{u}=\left(u_{1}, u_{2}, u_{3}\right)$ at the nodes of a regular array (grid). The changes of the void ratio $e$ are obtained from the equation

$\dot{e}=(1+e) \operatorname{tr} \mathbf{D}$

or

$\Delta e=(1+e)\left(\frac{\partial u_{1}}{\partial x_{1}}+\frac{\partial u_{2}}{\partial x_{2}}+\frac{\partial u_{3}}{\partial x_{3}}\right)$

The spatial derivatives are obtained by the linear approximation of the displacement vector field. They are affected by the number of points $n$ used in approximation and are unrealistically large on the free surface, adjacent to the tilting bar and on the slip surfaces where the displacement vector field changes abruptly. Therefore, a threshold $\chi$ is used to cut off too large derivatives: For any integers $i$ and $j$,

if $\left|u_{i, j}\right|>\chi$, then $u_{i, j}=0$

In addition, as $\Delta e$ is an approximation, considerable error arises while integrating it over time. For this reason, a factor $\eta$ is used to backscale it:

$e^{t+\Delta t}=e^{t}+\eta \Delta e$

Taking Test 1 as an example, the so obtained void ratios in the backfill after 10 cycles is shown Fig. 21. Improper selections of the three parameters $n, \chi$ and $\eta$ can lead to wrong estimation of $\Delta e$. For instance, Fig. 21a shows that void ratios pertain their initial values of 0.77 over 10 cycles, contradicting to the general agreement that the backfill becomes denser $[1,2]$. The other three combinations are shown in Figs. 21b, 21c and 21d. These results comply with the general agreement that the backfill gets denser over the cycles. Although the so obtained void ratios do not represent the exact ones, the tendency of their evolution provide valuable information on how density and/or pore space changes in such experiments. Fig. 22 shows the evolution of void ratio, using $n=49, \chi=0.002, \eta=0.7$, in cycles 5,10 , $15,25,35$, and 45 .

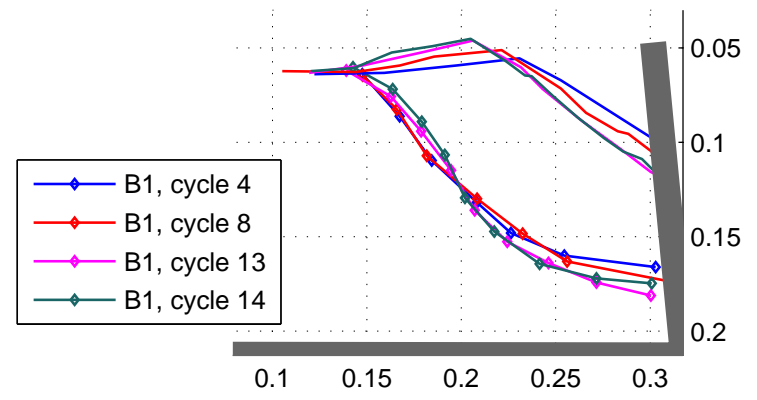

(a) B1-surface

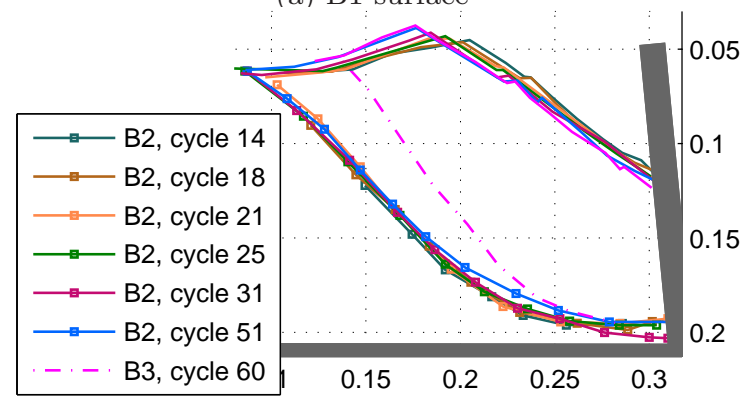

(b) B2-surface

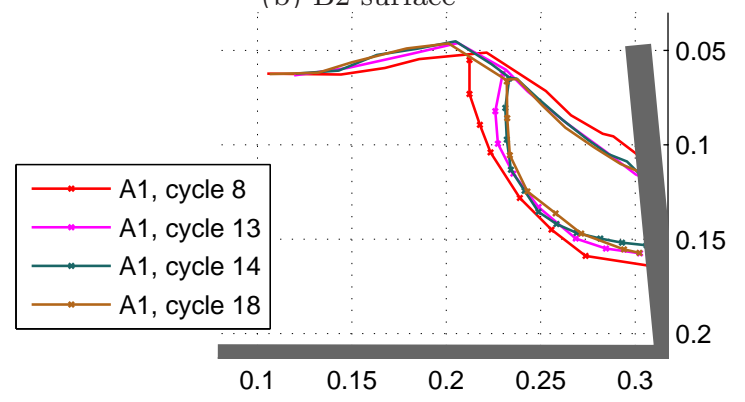

(c) A1-surface

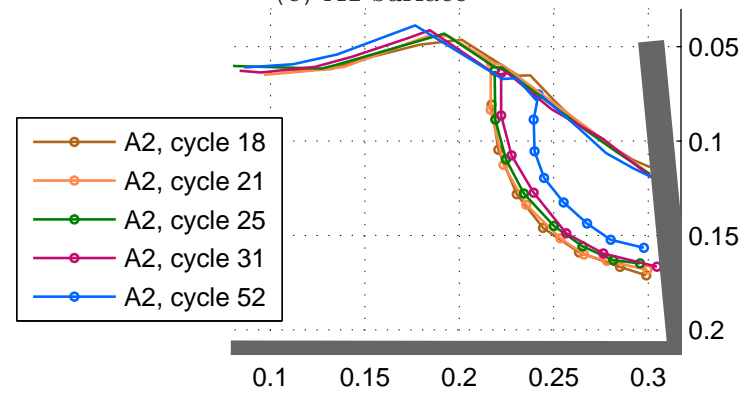

(d) A2-surface

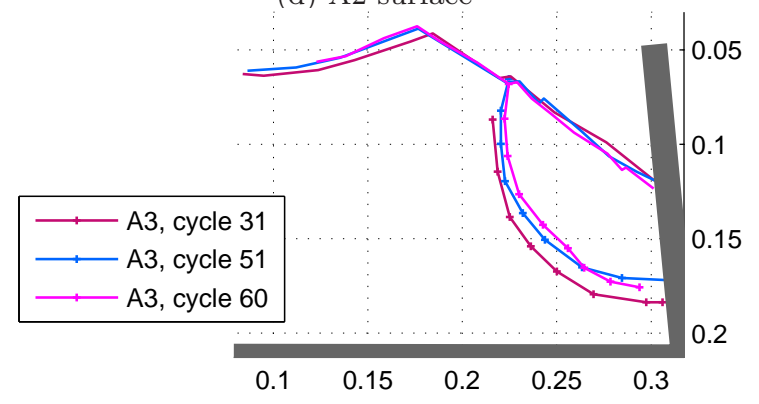

(e) A3-surface

Fig. 18: Evolution of passive slip surfaces in Test 1. Asurface and B-surface are denoted by A and B. Unit of axis in meter. 


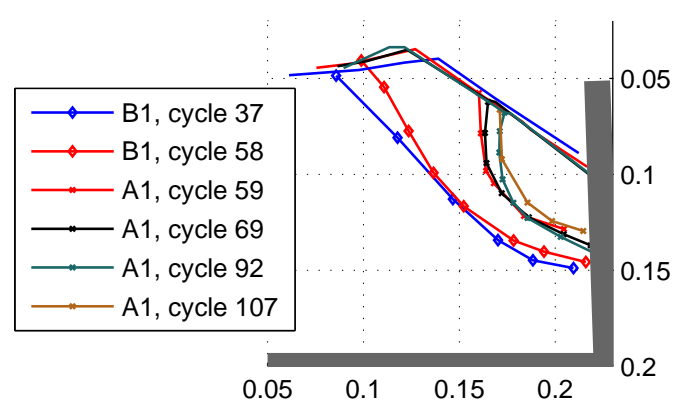

(a) A1- and A2-surface

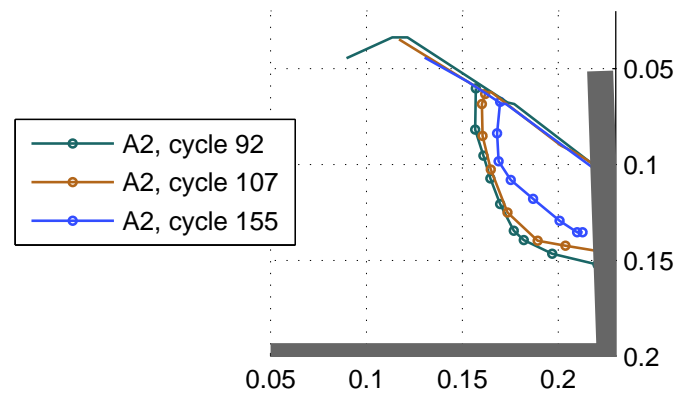

(b) Cycle 21 to the end

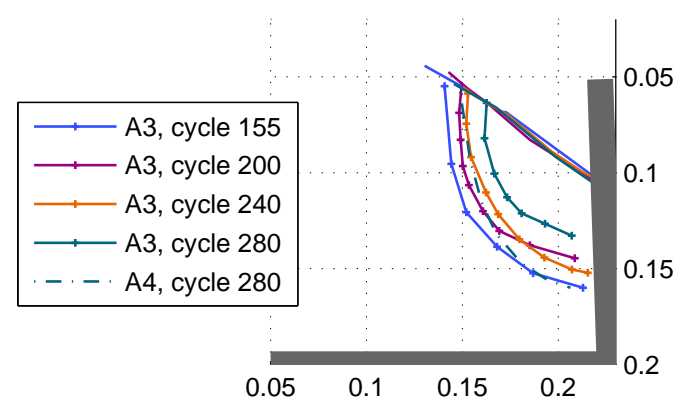

(c) Cycle 21 to the end

Fig. 19: Evolution of passive slip surfaces in Test 4. Asurface and B-surface are denoted by A and B

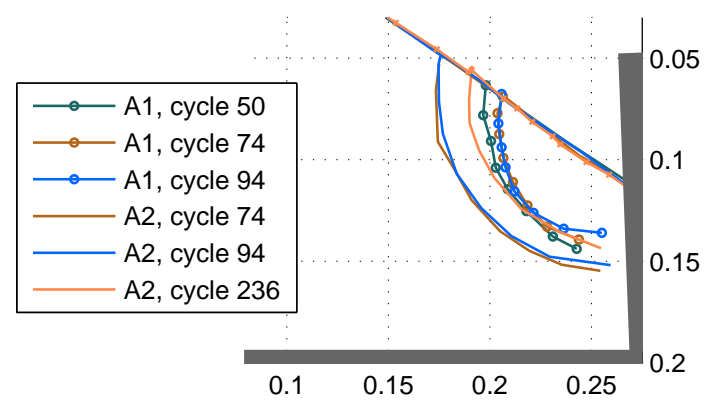

Fig. 20: Evolution of passive slip surfaces in Test 3. Asurface and B-surface are denoted by A and B.
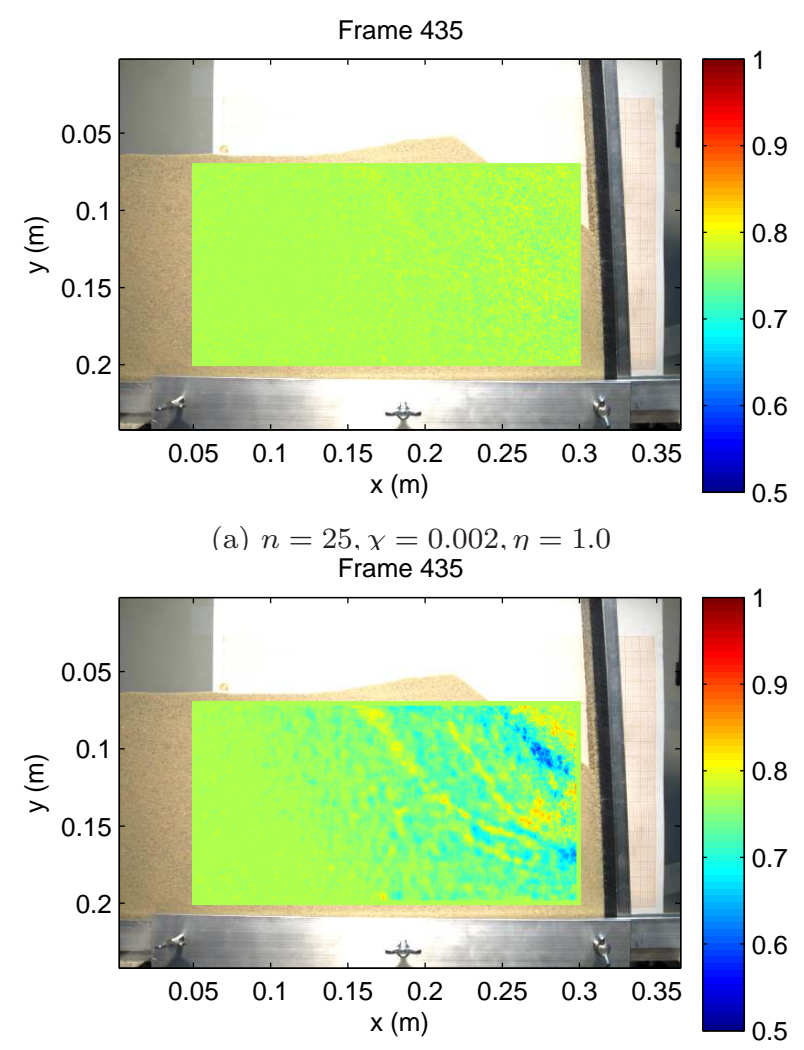

(b) $n=49, x=0.003, n=0.8$

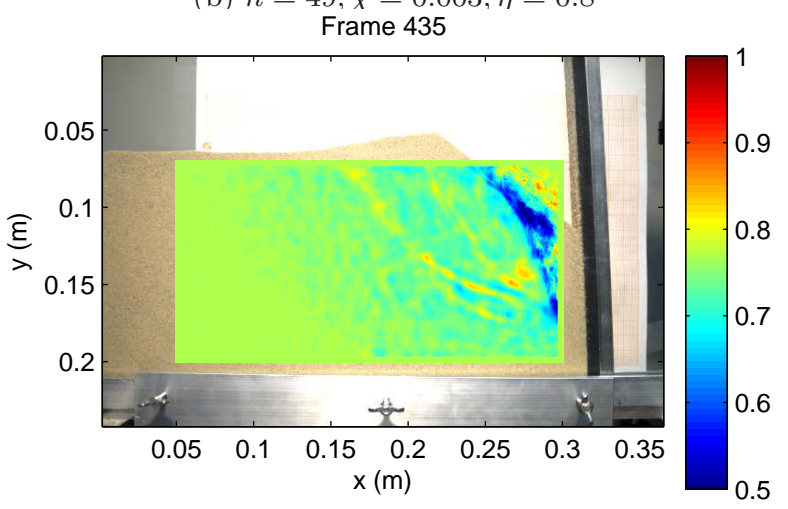

(c) $n=81, x=0.005, \eta=0.7$

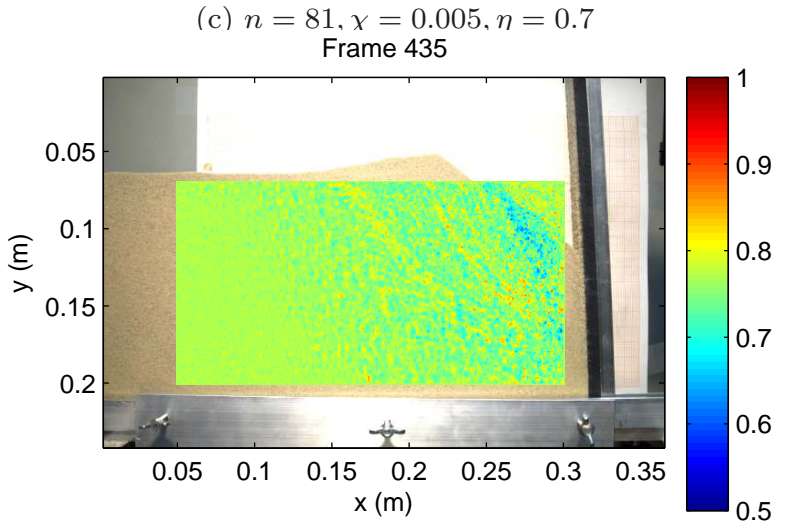

(d) $n=9, \chi=0.005, \eta=0.7$

Fig. 21: Void ratio in cycle 10 using various combinations of $n, \chi$ and $\eta$. 


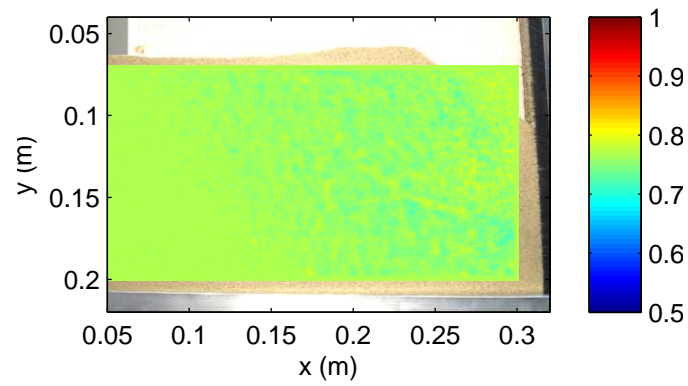

(a) Cycle 5

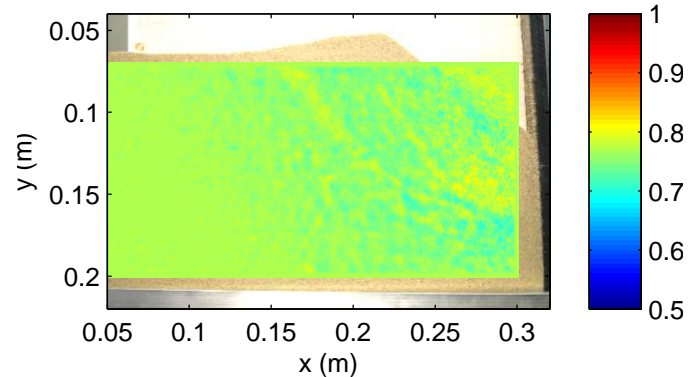

(b) Cycle 10

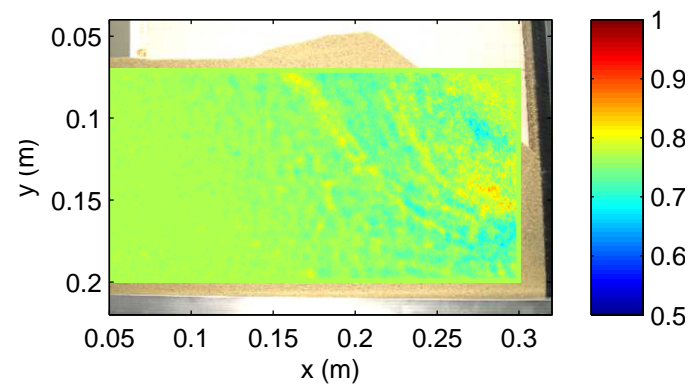

(c) Cycle 15

Fig. 22: Evolution of void ratio evaluated using $n=$ $49, \chi=0.002$ and $\eta=0.7$ in Test 1 , with initial void radio $e=0.77$. (continued)

These figures indicate that the backfill tends to become denser except of the passive slip surfaces, where shear bands appear (see Fig. 18 for comparisons). With increasing number of cycles, the density on the bottom of zone I decreases. This might result from the fact that the migration of the passive slip surfaces occurs in zone I.

\section{Conclusions}

The combination of the model test in plane strain conditions and the PIV technique makes it possible to capture the grain motion subject to cyclic tilt of a wall. Among all the findings presented in the previous sec-

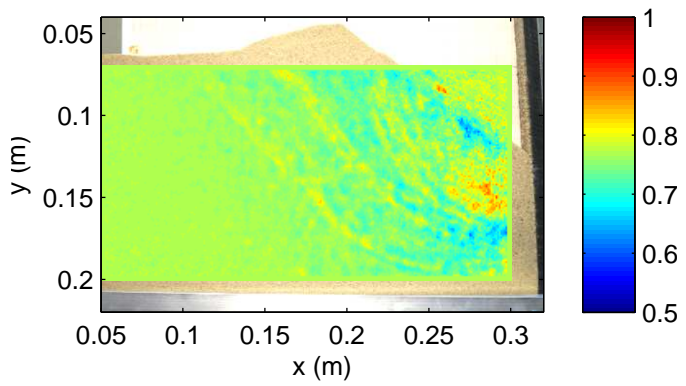

(d) Cycle 25

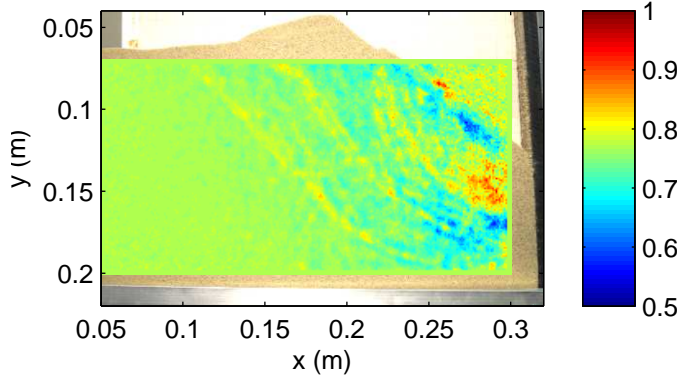

(e) Cycle 35

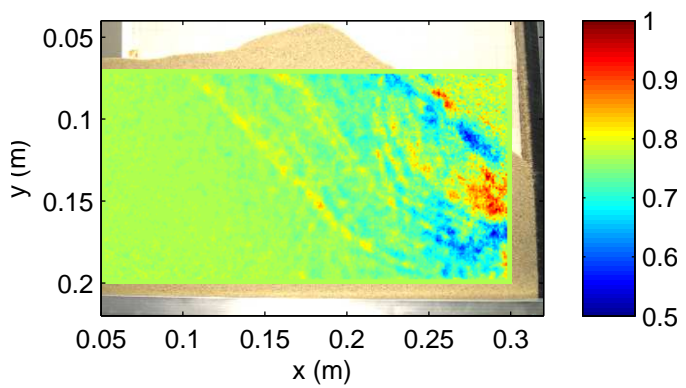

(f) Cycle 45

Fig. 22: (continued)

tions, the most remarkable ones are: (a) The visualization of the grain motion in terms of trajectories and velocity fields. (b) The understanding of the occurrence of eddies, namely the grains on the right side of the active slip surface exhibit the zigzag pattern in response to the cyclic tilt, moving to the lower right toward the wall until crossing the active slip surface to the other side, whereas those on the left side migrate to the upper left, end up with re-appearing on the surface or crossing the active slip surface. As a result of these two mechanisms, either closed or spiral non-smooth trajectories, i.e. sand eddies, occurred around the active slip surface. (c) Experiments with various tilt amplitudes and free surface configurations were carried out to study those effects. The width of domain II is found to be inversely proportional to the initial surface inclination angle, more 
cycles are required to form eddies of the same size if the tilt amplitude is smaller. (d) The evolution of void ratio was visualized. Besides all these findings, the experimental results themselves (e.g. the re-appearance migration of the passive slip surfaces) provide particlebased numerical simulation methods with challenging examples where a granular material undergoes large deformation and sophisticated mixing processes.

Acknowledgements The authors would like to thank the students Amy Cleaves and Eleanor Shirley for carrying out some of the experiments presented in this paper.

\section{References}

1. Bobryakov A.P., Kosykh V.P., and Revuzhenko A.F., "Temporary structures during the deformation of granular media, Fiziko-Tekhnicheskie Problemy Razrabotki Poleznykh Iskopaemykh", No. 1, pp. 29-39, March-April 1990, translated by Plenum Publishing Corporation.

2. Cuellar P., Maessler M., Ruecker W., "Ratcheting convective cells of sand grains around offshore piles under cyclic lateral loads", Granular Matter 11:379-390, 2009.

3. Thielicke W. and Stamhuis E. J. PIVlab - Time-Resolved Digital Particle Image Velocimetry Tool for MATLAB. Program downloadable at http://pivlab.blogspot.co.at/

4. Cheang, L., Matlock, H., "Static and cyclic lateral load tests on instrumented piles in sand." The Earth Technology Corporation, Long Beach, California, 1983. 